\title{
Evaluierung der Einzelserumdiagnose nach Influenza-Infektionen
}

\author{
Evaluation of Single Serum Diagnosis after Influenza Infections
}

Gabriele Döller, H.-J. Gerth

Abteilung für Medizinische Virologie und Epidemiologie der Viruskrankheiten, Hygiene-Institut, Tübingen

\begin{abstract}
Zusammenfassung:
Wir verglichen die diagnostische Wertigkeit der typspezifischen Komplementbindungsreaktion (KBR) mit einem typspezifischen indirekten Enzymimmuntest (ELISA) und einem subtypspezifischen indirekten Immunfluoreszenztest (IFT) in der serologischen Influenza-Diagnostik, wobei KBR und IFT, im Gegensatz zum ELISA, nicht automatisierbar sind. Die Eignung dieser Methoden zur Einzelserumdiagnose wurde ermittelt.

Grenztiter zur Erkennung einer frischen bzw. nur kurze Zeit zurückliegenden Influenza-Infektion wurden festgelegt. Die Spezifität wurde mit 103 Prä-Epidemieseren errechnet. Die Sensitivität wurde mit 62 Seren von 31 Patienten mit einer gesicherten Influenza-A-Infektion und mit 60 Seren von 30 Patienten mit einer gesicherten Influenza-B-Infektion ermittelt. Die Influenza-Infektionen waren durch einen signifikanten Titeranstieg in der KBR gesichert. Unter Berücksichtigung des oberen Grenztiters (Angaben in Klammern) wurden folgende Ergebnisse hinsichtlich Spezifität/Sensitivität erhalten: Influenza A: KBR (1: $\geq 80) 99 \% / 64 \%$, IFT-IgG (1: $\geq 2560)$

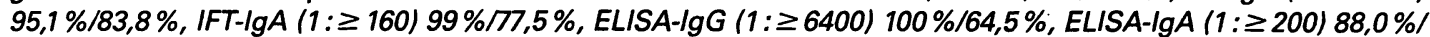
$81,5 \%$; und für Influenza B: KBR $(1: \geq 160) 100 \% / 72,5 \%$, IFT-IgG $(1: \geq 5120) 100 \% / 72,5 \%$, IFT-IgA $(1: \geq 160)$ $100 \% / 85,3 \%$, ELISA-IgG $(1: \geq 6400) 100 \% / 90,6 \%$, ELISA-IgA $(1: \geq 800) 96,1 \% / 87,9 \%$.
\end{abstract}

\section{Schlüsselwörter:}

Serodiagnose Influenza A - Serodiagnose Influenza B - KBR - IFT - ELISA - IgG-, IgA-, IgM-Antikörper - Subtyp-Spezifität - Typ-Spezifität

\section{Summary:}

We compared the diagnostic value of a type specific complement fixation test (CFT) with a type specific indirect enzyme immunoassay (ELISA) and a subtype specific indirect immunofluorescence test (IFT) in serological influenza diagnosis. In contrast to an ELISA, CFT and.IFT are not suitable for automation. The feasability of single serum diagnosis by these methods was examined. Borderline titers for recognition of acute or recent influenza infection were determined; specificity was calculated with 103 pre-epidemic sera. Sensitivity was determined by 62 sera of 31 patients with confirmed influenza $A$ infections and by 60 sera of 30 patients with confirmed influenza $B$ infections with different borderline titers. Using the upper borderline titers (in brackets) we obtained the following results in regard to specificity/sensitivity: Influenza A: CFT (1: $\geq 80) 99 \%$ / $64 \%$, IFT-IgG $(1: \geq 2560) 95,1 \% / 83,8 \%$, IFT-IgA $(1: \geq 160) 99 \% / 77,5 \%$, ELISA-IgG $(1: \geq 6400) 100 \% / 64,5 \%$, ELISA-IgA $(1: \geq 200) 88,0 \% / 81,5 \%$; and for influenza B: CFT $(1: \geq 160) 100 \% / 72,5 \%$, IFT-lg $(1: \geq 5120) 100 \%$ / $72,5 \%$, IFT-IgA $(1: \geq 160) 100 \% / 85,3 \%$, ELISA-IgG $(1: \geq 6400) 100 \% / 90,6 \%$, ELISA-IgA $(1: \geq 800) 96,1 \%$ / $87,9 \%$.

Keywords:

Serodiagnosis influenza $A$ - serodiagnosis influenza B - CFT - IFT-ELISA - IgG, IgA, IgM antibodies - subtype specificity - type specificity

\section{Einleitung}

Zur serologischen Diagnose einer Influenza werden auch heute noch in vielen Laboratorien die typspezifische Komplementbindungsreaktion (KBR) und/oder der stamm- bzw. subtypspezifische Hämagglutinationshemmtest (HHT) eingesetzt. Bei Vorliegen eines Serumpaares sind diese konventionellen Methoden zum Erkennen einer akuten bzw. kurze Zeit zurückliegenden Influenza-Infektion auch ausreichend. In der Praxis sind.jedoch Serumpaare oft nicht vorhanden bzw. Erstseren werden häufig erst sehr spät nach Auftreten der Krankheitssymptome abgenommen; so daß ein Titeranstieg nicht mehr festgestellt werden kann. Dennoch kann versucht werden, in diesen Fällen eine Verdachtsdiagnose mit einer Einzelserumúntersuchung zu bestätigen.

Wir berichteten bereits über den Vergleich eines subtypspezifischen Immunfluoreszenztests (IFT) mit der KBR in der Influenza-Diagnostik, wobei gezeigt werden konnte, daß der IFT bei Seren mit niedrigen, nur schwer interpretierbaren KBR-Titern $(1: 20$ oder $1: 40)$ hilfreich ist (Döller et al., 1986) $(1,2)$. Ein Nachteil beider Tests ist, daß sie nicht automatisiert werden können.

Ziel der vorliegenden Studie war die Evaluierung der diagnostischen Wertigkeit eines automatisierbaren typspezi- 


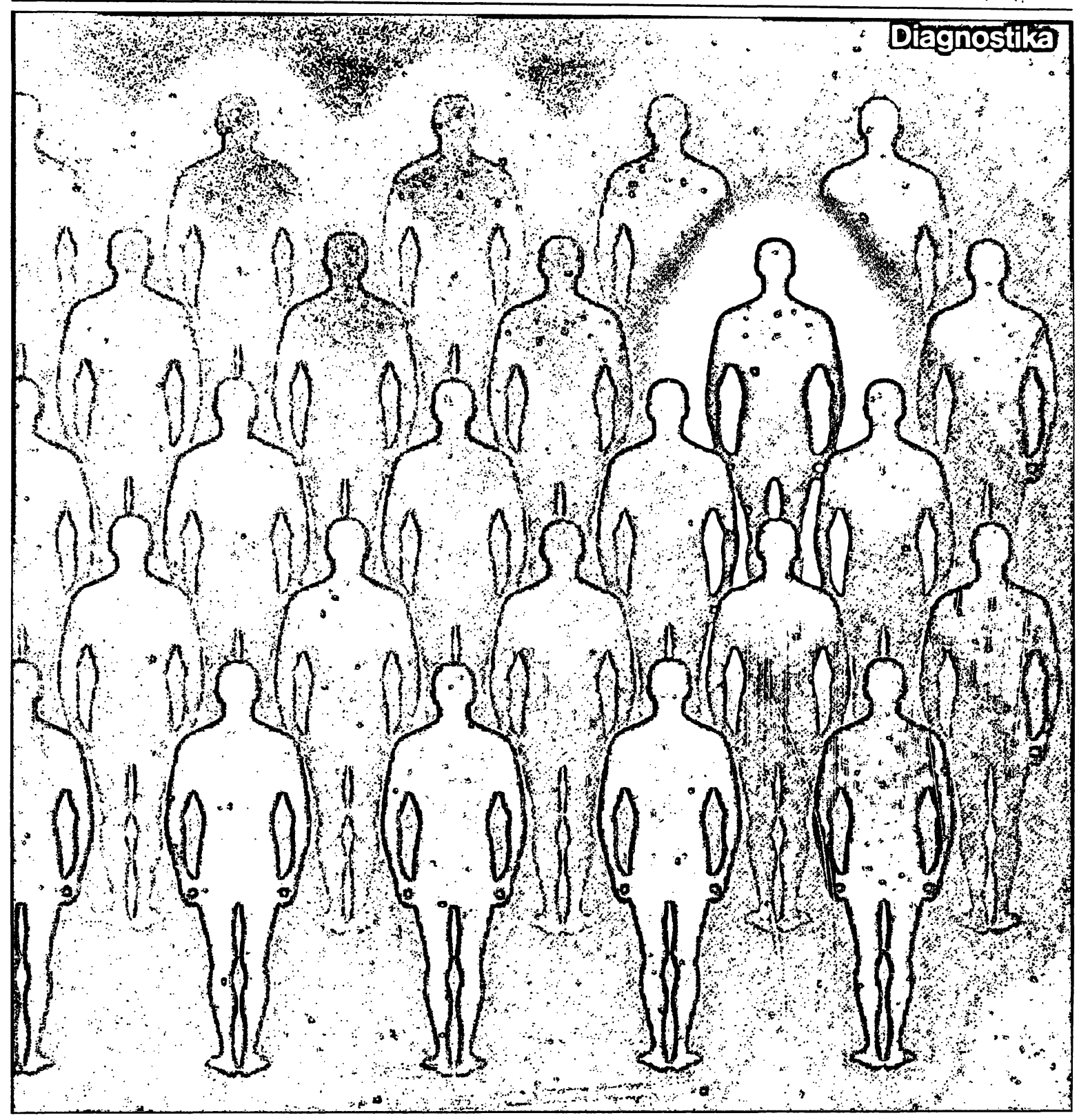

\section{Entdecken Sie den Unterschied. BETA-2-M-ELISA von medac.}




\section{Hämafologie-Systeme in der Praxis:}
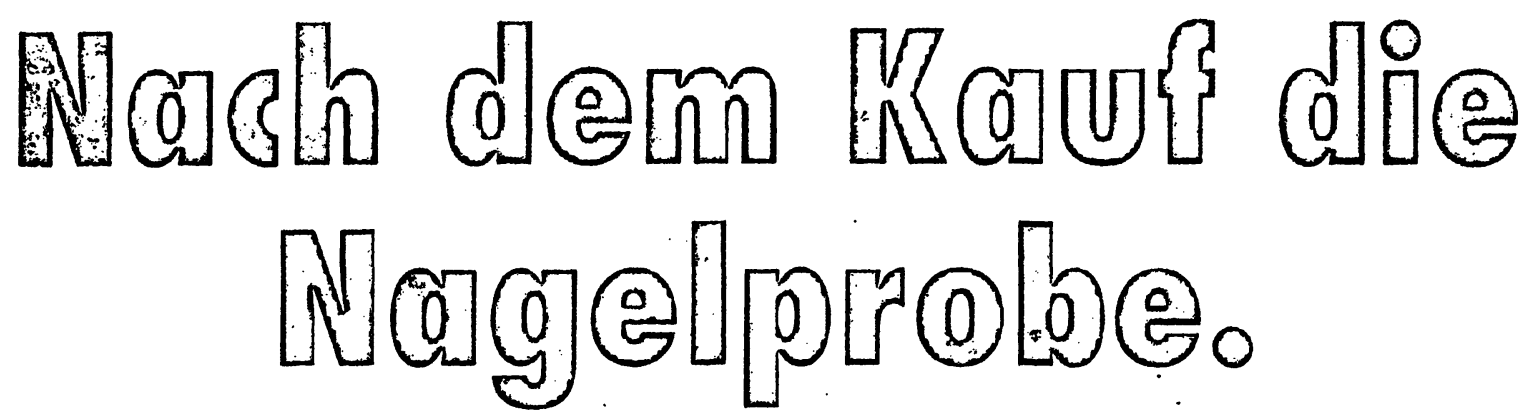

Hämatologie-Systeme sind keine Maschinen, die - einmal am Netz - praktisch wartungsfrei und unbeaufsichtigt arbeiten können. Es sind vielmehr komplexe Meßsysteme, die von Fachleuten bedient und von Fachleuten versorgt und gewartet werden müssen.

Darum endet auch eine Geschäftsbeziehung nicht mit dem Kauf eines Systems. Ganz im Gegenteil:

Hämatologie-Anwender wissen, daß sich die Qualität der Geschäftsbeziehung (und damit die Richtigkeit der Kaufentscheidung) erst nach dem Kauf erweist.

Stellen Sie daher rechtzeitig, d.h. vor dem Kauf, sicher, daß Sie danach nicht allein gelassen werden. Lassen Sie sich ein perfektes und bewährtes Service-System nachweisen, das Sie weder heute noch nach Jahren im Stich läßt.

Das ist Ihr gutes Recht. Fordern Sie es ein!

\section{Für die DIGITAMA ist diese Forderung Verpflichtung.}

- Mit dem Angebot von Sysmex Hämatologie-Systemen, deren Genauigkeit, Zuverlässigkeit und einfache Bedienung weltweit Maßstäbe gesetzt hat.

- Mit einem lückenlosen Angebot vom Basisgerät (Ery, Leuko, $\mathrm{Hb}$ ) bis hin zum Differenzier-Automaten und Flow-Zytometer.

- Mit einem dichten Netz von Service-und Beratungszentren in - Deutschland und der Schweiz.

- Mit einem Stab von System-Beratern, die - aus der Praxis kommend - Ihnen individuell beistehen und Sie streng praxisorientiert (nicht provisionsorientiert) informieren und beraten.

- Mit einer wissenschaftlichen Leitung, die Mitarbeiterschulung, Anwenderkurse und Weiterbildung anbietet und in engem Kontakt mit Standesorganisationen und medizinischen Fachgesellschaften Trends der Hämatologie mitbestimmt.

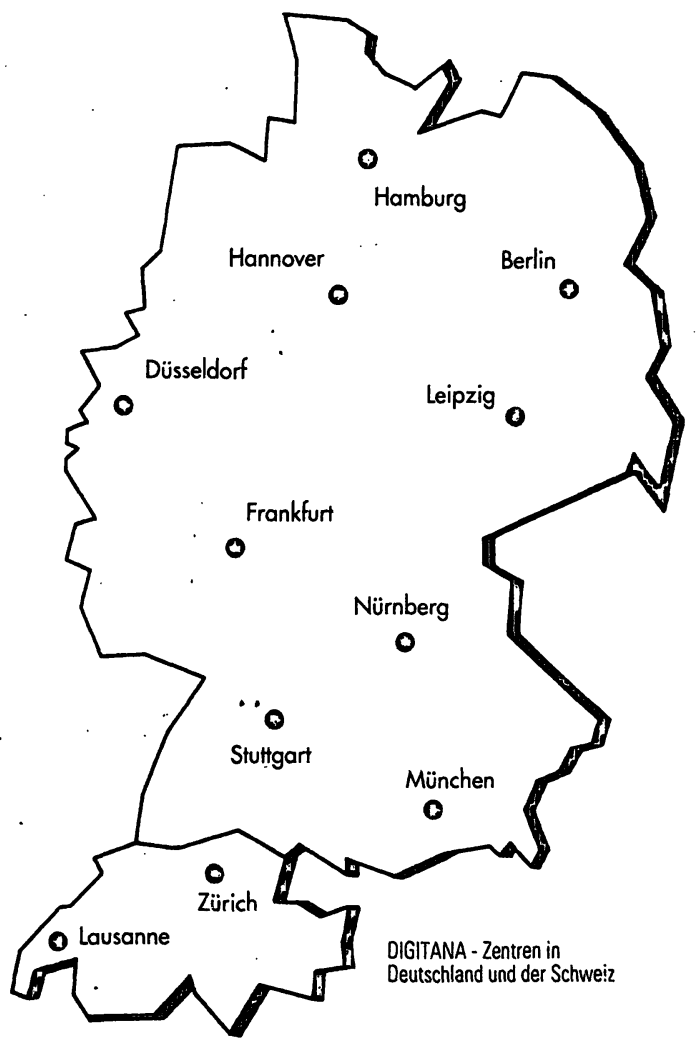

Damif beste Systeme auch in der Praxis Besfes leisten.

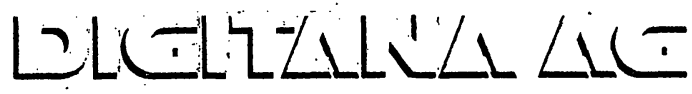

Ganz genau und zuverlässig.

0- 2000 Hamburg 76 - Weidestraße $118 \mathrm{~b}$. Tel:: $040 / 2707050$ $\mathrm{CH}-8810$ Horgen Burghaldenstraße 11. Tel.: $01 / 7256191$ 
fischen indirekten Enzymimmuntests (ELISA) im Vergleich zum IFT an Seren von Patienten, deren Influenzavirusinfektion klinisch wahrscheinlich war und die serologisch durch einen signifikanten Titeranstieg in der KBR bestätigt wurde.

\section{Material und Methoden}

\section{Enzymimmuntest}

$0,1 \mathrm{ml}$ Influenza-A- und -B- sowie Kontroll-Antigen für die KBR (Behringwerke AG, Marburg), 1:32 verdünnt in 0,001 $M$ Carbonatpuffer $(\mathrm{pH} 9,3)$ wurden in die Vertiefungen von Polystyrolchlorid-Mikrotiterplatten (Nunc, maxisorb plate; Nunc, Wiesbaden) gegeben und über Nacht bei $+4^{\circ} \mathrm{C}$ inkubiert. Die Absättigung der freien Bindungsstellen erfolgte durch Zusatz von $0,01 \mathrm{ml}$ Kälberserum pro Vertiefung über Nacht bei $+4^{\circ} \mathrm{C}$. Nach dreimaligem Waschen der Platten mit PBS (phosphate buffered saline: $\mathrm{pH} 7,2)$ erfolgte die Zugabe des zu testenden Humanserums, verdünnt in $5 \%$ (v/v) Kälberserum/PBS. Die Inkubationszeit betrug 2 Stunden bei $37^{\circ} \mathrm{C}$. Nach erneutem dreimaligen Waschen der Vertiefungen mit PBS erfolgte die Zugabe von $0,05 \mathrm{ml}$ Peroxidase markierten anti-humanIgG, -IgA oder -IgM (1:1000 [anti-human-lgG] bzw. 1:500 [anti-human-lgM und - IgA] verdünnt in $5 \%$ [v/v] Kälberserum $/ 5 \%$ [w/v] BSAPBS, Fa. DAKO, Vertrieb Boehringer, Ingelheim) pro Vertiefung. Die Inkubationszeit betrug 1 Stunde bei $37^{\circ} \mathrm{C}$. Anschließend wurden die Vertiefungen dreimal mit PBS gewaschen. Gebundene enzymmarkierte Antikörper wurden durch die Zugabe von 0,05 ml Substrat pro Vertiefung nachgewiesen. Als Substrat wurde $1 \mathrm{mg}$ 1,2-Phenylendiamin (Fa. Fluka, Buchs/ Schweiz) pro $\mathrm{ml} \mathrm{0,01} \mathrm{M} \mathrm{Phosphatpuffer} \mathrm{(pH} \mathrm{6)} \mathrm{unter} \mathrm{Zu}$ satz von $0,001 \% \mathrm{H}_{2} \mathrm{O}_{2}$ verwendet. Die Enzym-Substrat-Reaktion wurde mit $0,1 \mathrm{ml} \mathrm{H}_{2} \mathrm{SO}_{4}$ pro Vertiefung (IgG-ELISA: nach 4 Minuten; IgA-ELISA: nach 6 Minuten; IgM-ELISA: nach 8 Minuten) gestoppt.

Die Anfangsverdüninung der Seren betrug 1:100. Für den IgG-ELISA wurde in vierfachen Verdünnungsstufen titriert und für den $\lg A$ - und $\operatorname{Ig} M-E L I S A$ in zweifachen Verdünnungsstufen.

Der "Titer" wurde als die Serumverdünnung definiert, bei der noch eine OD-Differenz $\geq 0,2$ zwischen der Bindungsreaktion des zu testenden Serums mit Antigenund mit Kontrollantigen bei einer Wellenlänge von 490 nm (Dynatech MR 5000, Fa. Dynatech Denkendorf) bestand.

\section{Immunfluoreszenztest}

Für den Nachweis subtypspezifischer Antikörper wurde ein Immunfluoreszenztest verwendet, der bereits ausführlich beschrieben worden ist (Döller et al., 1985; Döller et al., 1986).

\section{Komplementbindungsreaktion}

Die Komplementbindungsreaktion (KBR) wurde mit kommerziell erhältlichem Influenza-A- und -B-Antigen für die KBR (Behringwerke AG, Marburg) nach einer Standardmikromethode durchgeführt (Lennette und Schmịdt, 1979).

\section{Patientenseren}

62 Seren von 31 Patienten, bei denen eine Influenza-A-Infektion durch einen signifikanten Titeranstieg in der KBR nachgewiesen worden war, und 60 Seren von 30 Patienten, bei denen eine Influenza-B-Infektion durch einen signifikanten Titeranstieg in der KBR nachgewiesen worden war, wurden vergleichend in der KBR, dem IFT und dem ELISA untersucht. Die Abnahme des Zweitserums
Tabelle: Berechnung der Spezifität und Sensitivität von KBR, IgG-IFT, IgA-IFT, IgG-ELISA und IgA-ELISA mit verschiedenen Grenztitern.

\begin{tabular}{lcccccc}
\hline Test & $\begin{array}{c}\text { rezip. } \\
\text { Titer* }\end{array}$ & $\begin{array}{c}\text { Spezi- } \\
\text { fität }\end{array}$ & $\begin{array}{c}\text { Sensi- } \\
\text { tivität }\end{array}$ & $\begin{array}{c}\text { rezip. } \\
\text { Titer** }\end{array}$ & $\begin{array}{c}\text { Spezi- } \\
\text { fität }\end{array}$ & $\begin{array}{c}\text { Sensi- } \\
\text { tivität }\end{array}$ \\
\hline Influenza A: & & $(n=103)$ & $(n=62)$ & & $(n=103)$ & $(n=62)$ \\
KBR: & $\geq 80$ & $99,0 \%$ & $64,0 \%$ & $\geq 40$ & $88,0 \%$ & $81,5 \%$ \\
IFT-IgG: & $\geq 2560$ & $95,1 \%$ & $83,8 \%$ & $\geq 1280$ & $59,2 \%$ & $96,9 \%$ \\
IFT-IgA: & $\geq 160$ & $99,0 \%$ & $77,5 \%$ & $\geq 80$ & $97,1 \%$ & $79,4 \%$ \\
ELISA-lgG: & $\geq 6400$ & $100,0 \%$ & $64,5 \%$ & $\geq 1600$ & $100,0 \%$ & $91,1 \%$ \\
ELISA-IgA: & $\geq 200$ & $88,0 \%$ & $81,5 \%$ & $\geq 100$ & $88,0 \%$ & $91,1 \%$ \\
Influenza B: & & $(n=103)$ & $(n=60)$ & & $(n=103)$ & $(n=60)$ \\
KBR: & $\geq 160$ & $100,0 \%$ & $72,5 \%$ & $\geq 80$ & $96,1 \%$ & $78,4 \%$ \\
IFT-IgG: & $\geq 5120$ & $100,0 \%$ & $72,5 \%$ & $\geq 2560$ & $95,1 \%$ & $90,6 \%$ \\
IFT-IgA: & $\geq 160$ & $100,0 \%$ & $85,3 \%$ & $\geq 80$ & $90,3 \%$ & $90,6 \%$ \\
ELISA-lgG: & $\geq 6400$ & $100,0 \%$ & $90,6 \%$ & $\geq 1600$ & $99,0 \%$ & $96,7 \%$ \\
ELISA-lgA: & $\geq 800$ & $96,1 \%$ & $87,9 \%$ & $\geq 400$ & $96,1 \%$ & $87,9 \%$ \\
\hline
\end{tabular}

rezip. Titer* = oberer Grenztiter

rezip. Titer" $=$ unterer Grenztiter

erfolgte zwischen Tag 5 und 31 nach der Erstserumabnahme. Die Seren waren zwischen 1983 und 1991 gesammelt worden und entweder sofort nach Eintreffen im Laboratorium untersucht oder bei $-20^{\circ} \mathrm{C}$ bis zur Untersuchung gelagert worden.

Für die Bestimmung der Spezifität nach Galen und Gambino (1979) wurden 103 Blutspenderseren untersucht. Die Seren sind im Januar 1985 vor Beginn der damaligen Influenza-Aktivität abgenommen worden.

\section{Ergebnisse}

\section{Ermittlung der Sensitivität und Spezifität}

Die Ergebnisse der Sensitivitäts- und Spezifitätsberechnung für einen oberen und einen unteren "Grenztiter", der auf eine kurze Zeit zurückliegende Influenzavirusinfektion hindeutet, sind in der Tabelle dargestellt.

Aus der Tabelle ist zu entnehmen, daß zum Nachweis von Antikörpern gegen Influenza A der IFT-IgG mit einer Sensitivität von $83,8 \%$ (oberer Grenztiter) rsp. $96,9 \%$ (unterer Grenztiter) die besten Ergebnisse zeigte, hingegen war der Nachweis von Antikörpern gegen Influenza B mit dem ELISA-lgG mit einer Sensitivität von $90,6 \%$ (oberer Grenztiter) rsp. $96,7 \%$ (unterer Grenztiter) am zuverlässigsten. In allen Testsystemen wurde ein Spezifitätsverlust von 0 bis $1.1 \%$ zwischen den beiden Grenztitern beobachtet mit Ausnahme des Influenza-A-IgG-IFT, der mit dem oberen Grenztiter eine Spezifität von $95,1 \%$ und mit dem unteren Grenztiter eine Spezifität von nur $59,2 \%$ aufzeigte.

\section{Diskussion}

Im allgemeinen ist die Einzelserumdiagnose bei häufig vorkommenden viralen Erkrankungen von einer guten Information von seiten des behandelnden Arztes abhängig. Wichtige bènötigte Informationen wie Tag des Erkrankungsbeginns mit klinischen Erscheinungsbildern sind für die Interpretation des Befundes bei einer Einzelserumdiagnose unerläßlich. Innerhalb dieser Studie wurde die diagnostische Wertigkeit einer typ- bzw. subtypspezifischen Immunantwort nach natürlicher Influenza-Infektion ermittelt. Ziel der Studie war es, die Effektivität von KBR, ELISA und IFT bei einer Einzelserumdiagnose zu evaluieren. Dazu wurden die Grenztiter, die auf eine akute oder kürzlich stattgefundene Influenza-Infektion hindeuten, unter Berücksichtigung der Titer von Prä-Epidemiese- 
ren angenommen. IgM-Antikörper wurden nicht berücksichtigt, da von mehreren Autoren nur unregelmäßiges Auftreten von spezifischen IgM-Antikörpern nach Influenza-Infektion beschrieben worden war (Julkunen et al., 1985; Harmon et al., 1986; Koskinen et al., 1987). Bei allen Influenza-A-Patienten war innerhalb dieser Studie mit dem IFT eine Influenza AH3N2-Infektion diagnostiziert worden.

Unsere Ergebnisse zeigen, daß für die serologische Diagnose einer Influenza-A-Infektion der IgG-IFT mit einer Sensitivität von $83,8 \%$ rsp. $96,9 \%$ bei einer Einzelserumdiagnose innerhalb von 31 Tagen nach Auftreten der ersten Krankheitszeichen am geeignetsten war.

Überraschenderweise war für die Diagnose einer Influenza-A-Infektion der IgG-ELISA bei Verwendung des oberen Grenztiters der KBR nur geringfügig überlegen. Diese Ergebnisse stehen im Gegensatz zu bisher erschienenen Arbeiten (Julkunen et al., 1985; Masihi und Lange, 1980; Joassin et al., 1983). Hier muß jedoch berücksichtigt werden, daß wir aus Praktikabilitätsgründen für den IgGELISA vierfache Verdünnungsstufen ausgewählt hatten. Die Anwendung einer Verdünnung von z. B. 1:3200 hätte mit großer Wahrscheinlichkeit bessere Ergebnisse erbracht.

Im Gegensatz zu den Ergebnissen, die wir für den Nachweis von Antikörpern gegen Influenza-A-Virus erhalten haben, ist der Influenza-B-IgG-ELISA mit einer Sensitivität von $90,6 \%$ rsp. $96,7 \%$ allen anderen Testsystemen überlegen. Die schlechtesten Ergebnisse wurden mit der KBR und dem IgG-IFT erhalten. Ähnliche Beobachtungen bezüglich der KBR sind auch von anderen Autoren beschrieben worden (Julkunen et al., 1985).

\section{Standardisierte Reagenzien für mikrobiologische Laboratoriums-Arbeiten}

dehydrierte sowie Fertigmedien, Gewebezuchtmaterialien, Seren und Reagenzien

Resistenz-Blättchen, Carbohydrate, Farbstoffe, Antigene und Seren, Dispenser, FA-Reagenzien, Peptone, Hydrolysate, Indikatoren, Exirakłe, Biochemikalien, Aminosäuren, Enzyme, serolog. Teste sowie klinische Reagenzien und Anreicherungsmedien.

\section{DIFCO-Produkte werden routinemäßig in

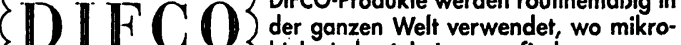 I. I biologische Arbeiten stattfinden.}

Sie wurden speziell geschaffen für die klin.-chem. Laboratorien in Krankenhäusern. Hygiene-Institute, GewebezuchtLaboratorien, Gesundheitsämter - für Wasser- und Abwasserkontrollen, Milch- und Lebensmitteluntersuchungen, Qualitätsüberwachung in der chem.-pharm. Industrie, Erziehungs- und Ausbildungsinstitute.

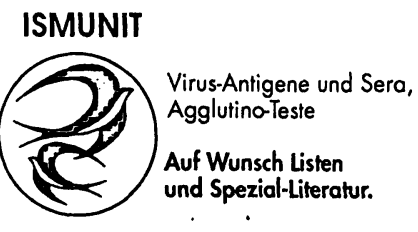

Heinrichstraße $5 \cdot 2000$ Hamburg 50 Tel. 040/432827. Fax 040/432830
Die Spezifität aller Testsysteme war erwartungsgemäß höher bei Verwendung des oberen Grenztiters im Gegensatz zu dem unteren Grenztiter. Nur in einem Testsystem (Influenza A/H3N2-IFT-IgG) war eine große Differenz zwischen den Spezifitäten beider Grenztiter beobachtet worden. Dies ist das einzige Testsystem, bei dem nur eine einzige Titerstufe $11: 1280$ : niedriger Grenztiter; $1: 2560$ : hoher Grenztiter) von Bedeutung für die Diagnose einer kürzlich stattgefundenen Influenza-A-Infektion ist.

Aufgrund der Ergebnisse zogen wir den Schluß, daß die Einzelserumdiagnose einer Influenza-A-Infektion mit dem IgG-IFT und einer Influenza-B-Infektion mit dem IgGELISA mit relativ großer Treffsicherheit möglich ist.

Schriftum:

1. DOLLER, G., DOLLER, P. C., GERTH, H.-J.: Diagnostic significance of influenza subtype-specific IgG, IgA, and IgM antibodies. J. Biol. Stand. 14, 163-175 (1986).

2. DOLLER, G., DOLLER, P. C., GERTH, H.-J.: Serologische Diagnostik von Influenza Aund B-Infektionen mit Hilfe der Komplementbindungsreaktion und der indirekten Immunfluoreszenztechnik. Lab.med. 10, 291-296 (1986).

3. DOLLER, P. C., DOLLER, G., GERTH, H.-J.: Immunofluorescence test with antigen loaded erythrocytes: detection of influenza virus specific $\lg G, \lg A$, and $\lg M$ antibodies. Med. Microbiol. Immunol. 173, 291-302 (1985).

4. GALEN, R. S., GAMBINO, S. R.: Norm und Normabweichung klinischer Daten. G. Fischer Verlag. Stuttgart-New York, 85 (1985).

5. HARMON, M. W., PHILLIPS, D. J., REIMER, C. B., KENDAL, A. P.: Isotype-specific immunoassay for influenza antibody with monoclonal antibodies to human immunoglobulins. J. Clin. Microbiol. 24, 913-916 (1986).

6. JOASSIN, L., REGINSTER, M., VAIRA, D.: Anti M-protein antibody response to type $A$ or $B$ natural influenza detected by solid phase enzyme linked immunosorbent assay and by complement fixation. Arch. Virol. 76, 15-23 (1983).

7. JULKUNEN, I., PYHALLA, R., HOVI, T.: Enzyme immunoassay, complement fixation and hemagglutination inhibition tests in the diagnosis of influenza $A$ and $B$ virus infections. Purified hemagglutinin in subtype-specific diagnosis. J. Virol. Meth. 10, 75-84 (1985)

8. KOSKINEN, P., VUORINEN, T., MEURMAN, O.: Influenza A and B virus IgG and IgM serology by enzyme immunoassays. Epidem. Inf. 99, 55-64 (1987).

9. LENNETTE, E. H., SCHMIDT, N. J.: Complement-fixation (CF) test. In: LENNETTE, E. H., SCHMIDT, N. J. (eds.). Diagnostic Procedures for Viral, Rickettsial and Chlamydial Infections. 5th Edition. Washington DC, American Public Health Association, 35-42 (1979).

10. MASIHI, K., LANGE, W.: Enzyme-linked immunosorbent assay for the detection of influenza type-specific antibodies. J. Immunol. Meth. 36, 173-179 (1980).

\section{Acknowledgement}

Wir danken Edeltraud Faigle und Monika Kuhar für ausgezeichnete technische Assistenz. Die Arbeit wurde durch das Bundesministerium für Forschung und Technologie unterstützt (01ZR87052). Die Verantwortung für den Inhalt der Arbeit liegt bei den Autoren.

\author{
Anschrift der Verfasser: \\ Dr. Gabriele Döller \\ Prof. Dr. H.-J. Gerth \\ Hygiene-Institut \\ Abt. f. Med. Virologie u. Epidemiologie der Viruskrankheiten \\ Silcherstraße 7 \\ 7400 Tübingen
}




\section{DMAENSION SMS}

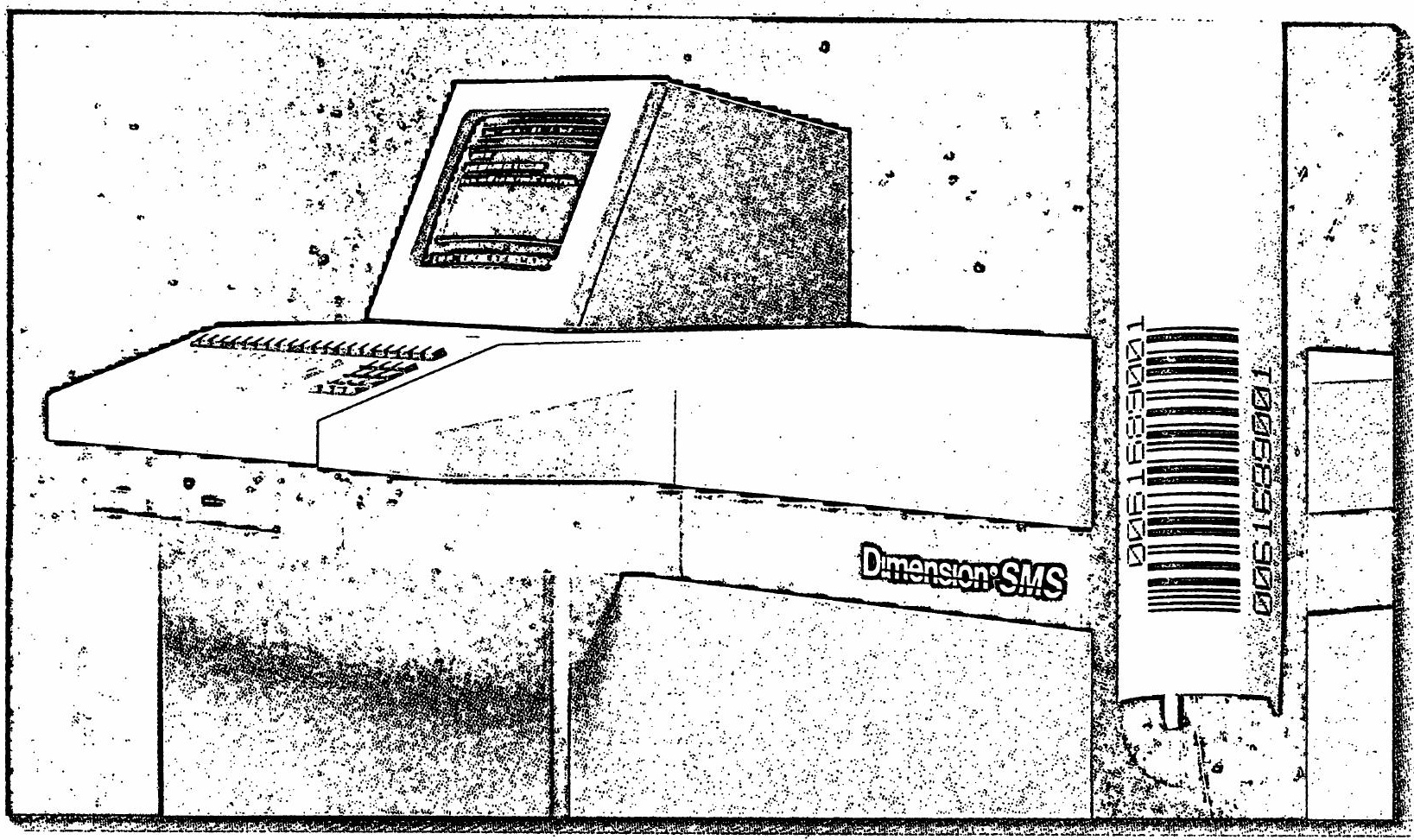

\section{Immer ein Schritt voraus:}

... flexibel, zuverlässig, einfach

Große Flexibilität bietet das neue System durch zehn frei programmierbare Kanäle. Für Bestimmungen, die Du Pont zur Zeit noch nicht anbietet, können problemlos Reagenzien Ihrer Wahl verwendet werden.

Positive Probenidentifikation auf unterschiedlichen Primärgefäßen bei einfachster Handhabung kennzeichnen den Dimension ${ }^{\circledR}$ SMS. Sie können jederzeit jede Probe bearbeiten, Notfälle einschieben und Kontrollen fahren - ohne Zeitverlust, ohne Umrüsten.

\section{Produktbereich Diagnostika}

39 Methoden decken mehr als $90 \%$ der Testanforderungen in der Routine ab.

Hohe Zuverlässigkeit in der Datenerfassung bringt der neuartige Barcode-Leser. Alle gängigen Barcodes können verwendet werden.

Wann sehen Sie sich Ihren Dimension ${ }^{\circledR}$ SMS näher ạn? Anruf genügt!

Wir führen Ihnen das System gerne vor. Tel.: 06172 / 872535

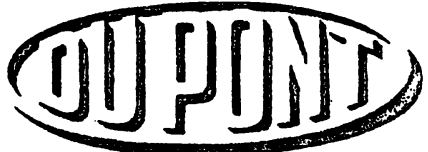




\section{Unsere Produlkse fiư⿱宀 oflie

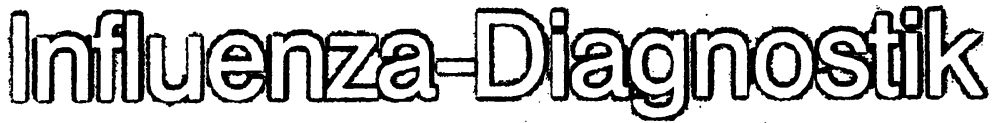
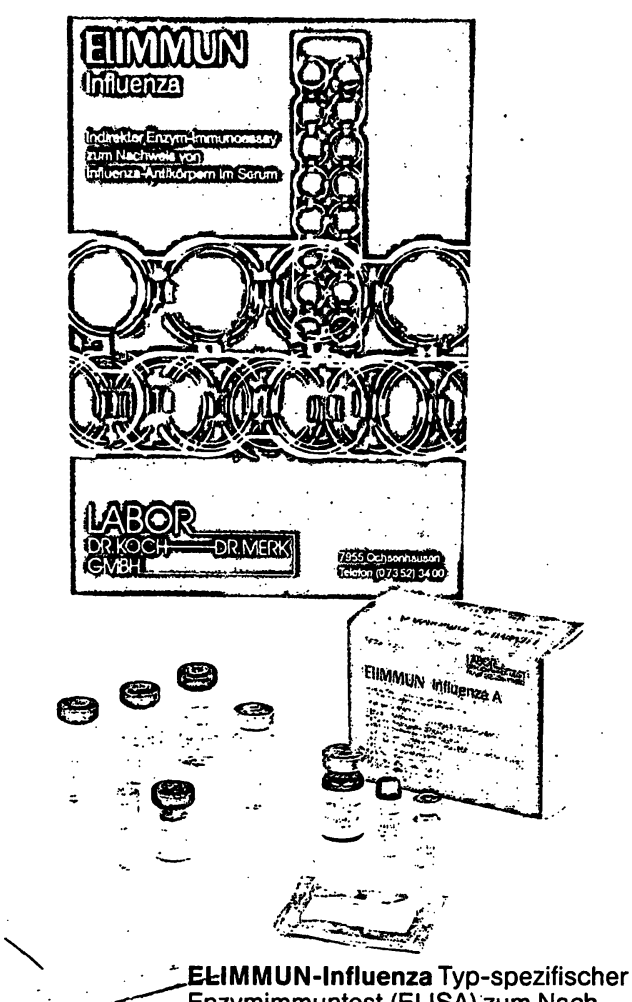
Enzymimmuntest (ELISA) zum Nachweis von IgG- und IgA-Antikörpern gegen Influenza A und $B$

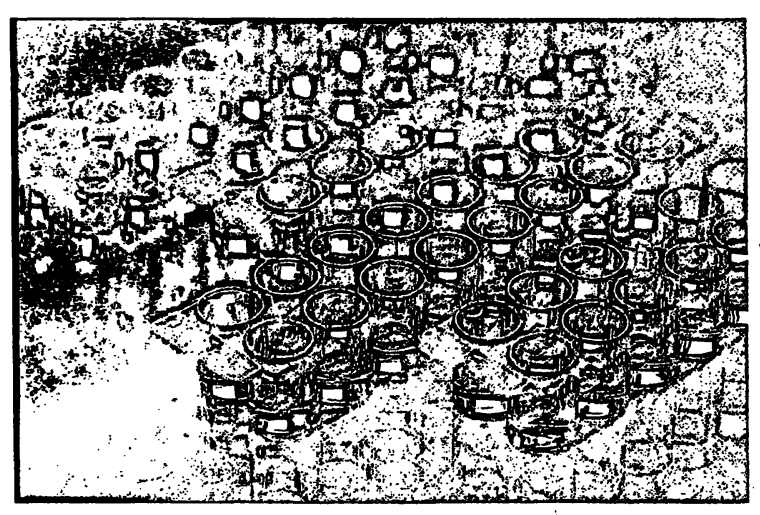

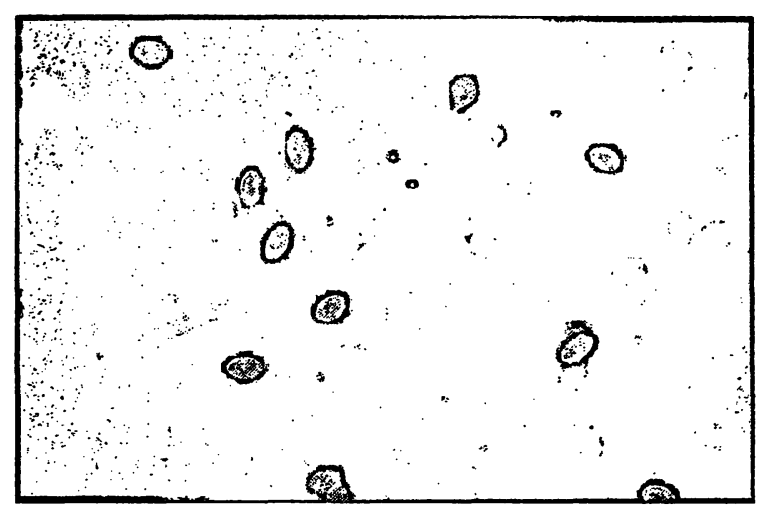

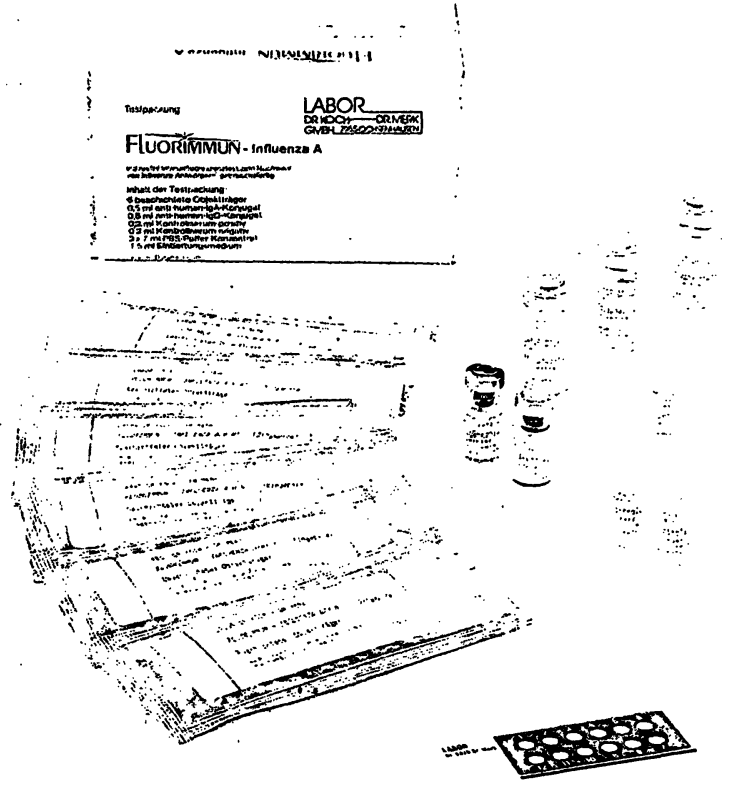

FLUORIMMUN-Influenza

Typ-oder Subtyp-spezifischer indirekter Immunfluoreszenztest (IFT) zum Nachweis von.lgG- und lgA-Antikörpern gegen Influenza A, bzw. A/Subtyp H1/N1 oder Subtyp H3N2 und Influenza $B$.

\section{D-7955 Ochsenhausen} Schloßstraße 9

\section{$\angle A B O R$}

DR.KOCH DR.MÉRK $\mathrm{GMBH}$
Telefon (07352) 3400

Telefax (07352) 4834 